1013

\title{
Exploring Trends in Microcrack Properties of Shales Using Stress Dependencies of Ultrasonic Velocities
}

\author{
M. Pervukhina* (CSIRO), B. Gurevich (Curtin University of Technology/ \\ CSIRO) \& P. Golodoniuc (Curtin University of Technology/CSIRO)
}

\section{SUMMARY}

Stress dependency of full elastic tensor of shales is very important for seismic interpretation, overpressure prediction, 4D monitoring, etc. Using Sayers-Kachanov formalism, we develop a new stress sensitivity model for transversely isotropic (TI) media which predicts stress sensitivity behaviour of all five elastic constants. The model is used to parameterize elastic properties of about 20 shales obtained by laboratory measurements and from literature survey. The four fitting parameters (namely, tangential and normal compliances of a single crack, characteristic pressure, and pore orientation anisotropy parameter) show obvious correlations with the depth from which the shale was extracted. With increase of the depth, the tangential compliance decreases exponentially and the ratio of normal to tangential compliance increases linearly. The crack orientation anisotropy parameter exponentially increases with the depth for the most of the shales indicating that cracks are getting more aligned in the bedding plane. The characteristic pressure shows no visible correlation with the depth. The suggested model allows predicting of stress dependency of all five elastic constants if only two of them are known what can be useful, for instance, for the reconstruction of all five elastic constants of shale from log data. 


\section{Introduction}

Stress dependency of full elastic tensor of shales is practically important for seismic interpretation, overpressure prediction, 4D monitoring, etc. In spite of the importance of the problem, there is no conventional theory which allows describing stress dependency of shale elastic properties with a small number of physically plausible parameters. Shapiro and Kaselow (2005) suggested a stress dependency model for orthorhombic media based on dual porosity approach that imply bimodal distribution of pore compliances and superposition of deformation fields caused by closure/shape change of these two group of pores under applied stress. Assumption that was made by Shapiro and Kaselow (2005) and Ciz and Shapiro (2009) to apply this dual porosity model to transversely isotropic (TI) media implicitly assumes that normal and tangential compliances of each individual crack are equal. This assumption oversimplifies the model and does not allow prediction of stress dependencies of all five elastic constants of TI media, i.e. $\mathrm{c}_{13}$ elastic constant does not depend on hydrostatic stress (Shapiro and Kaselow, 2005; Ciz and Shapiro, 2009). Here we use formalism suggested by Sayers and Kachanov (1995) to develop a model that describes stress dependencies of all five elastic constants of TI media.

\section{Model}

The suggested model of stress dependency of elastic properties of shale assumes that variation of elastic properties with pressure up to $60 \mathrm{MPa}$ can be explained with closure of soft (compliant) porosity. Soft porosity comprises a small part of total porosity and consists of pores with high compliances, for instance, fractures, cracks and grain contacts. Figure 1 shows an SEM image which illustrates existence of such type of pores in shales.

Sayers and Kachanov (1995) provided a formalism that allows calculation of an excess of compliances $\Delta S_{i j k l}$ due to the introduction of the compliant porosity to a rock matrix with compliance of $S_{i j k l}$ as follows,

$$
\begin{aligned}
& \Delta S_{i j k l} \equiv S_{i j k l}-S_{i j k l}^{0}=\frac{1}{4}\left(\delta_{i k} \alpha_{j l}+\delta_{i l} \alpha_{j k}+\delta_{j k} \alpha_{i l}+\delta_{j l} \alpha_{i k}\right)+\beta_{i j k l} \\
& \alpha_{i j}=\frac{1}{V} \sum_{r} B_{T}^{(r)} n_{i}^{(r)} n_{j}^{(r)} A^{(r)} \\
& \beta_{i j k l}=\frac{1}{V} \sum_{r}\left(B_{N}^{(r)}-B_{T}^{(r)} n_{i}^{(r)} n_{j}^{(r)} n_{k}^{(r)} n_{l}^{(r)} A^{(r)} .\right.
\end{aligned}
$$

Here, $\delta_{i j}$ is the Kronnecker delta, $r$ is the number of planar discontinuities with surface area $A^{(r)}$ and surface-normal vectors $n_{i}^{(r)}$ in volume $V$ and $B_{N}$ and $B_{T}$ are the normal and tangential compliances of an individual crack.

To parameterise the stress dependency of shales, we use four parameters, namely, $B_{T}$, tangential compliance, $B$, ratio of normal to tangential compliance, $N$, normalized number of crack with normal vector forming angle $\theta$ with vertical direction and $P$, characteristic pressure. Number of cracks with particular orientation is determined as $N(\theta)=1+\eta \cos ^{2} \theta$, where $\eta$ is crack orientation anisotropy parameter. We suppose that tangential compliance decreases exponentially with pressure as follows $B_{T}=B_{T 0} \exp \left(-P / P_{0}\right)$. Substituting these formulas into equations (1-3), we obtain variation in compliances introduced by the compliant porosity.

$$
\Delta S_{11}=\frac{B_{T 0} \exp \left(-P / P_{0}\right)}{105}(14+4 \eta+21 B+3 B \eta)
$$




$$
\begin{aligned}
\Delta S_{33} & =\frac{B_{T 0} \exp \left(-P / P_{0}\right)}{105}(14+6 \eta+21 B+15 B \eta) \\
\Delta S_{44} & =\frac{B_{T 0} \exp \left(-P / P_{0}\right)}{105}(42+16 \eta+28 B+12 B \eta) \\
\Delta S_{66} & =\frac{B_{T 0} \exp \left(-P / P_{0}\right)}{105}(42+10 \eta+28 B+4 B \eta) \\
\Delta S_{13} & =\frac{B_{T 0} \exp \left(-P / P_{0}\right)}{105}(7 B+3 B \eta-7-3 \eta) .
\end{aligned}
$$

\section{Data}

We used both new and published experimental results (Wang, 2002) in current study. Shale samples used in this paper are shales from the Officer Basin, the Bass Basin, the Carnarvon Basin, Africa shales, North Sea shales (Pervukhina et al, 2008), Gulf Coast shale, Hard shales and siliceous shale (Wang, 2002). The shales were extracted from the depths ranging from 200 to $3604 \mathrm{~m}$ and vary in their physical properties and, most probably, in their mineralogical content. Unfortunately, only few publications report information about overbudern pressure, diagenesis history, clay content and mineralogy of the investigated shales. The depth of origin of the shale samples is the only parameter known for all the shales.

\section{Results}

We use equations (4-8) to fit experimentally obtained stress dependencies of elastic constants of shales considering $B_{T 0}, B, P_{0}$ and $\eta$ as fitting parameters. Histogram of the ratio of tangential over normal compliances, $B$, obtained by the fitting is shown in Figure 2. The values are distributed in three distinct groups, namely, small values less than 0.2 , the normal values from 0.7-1.1 and the large values of about 2 . The strong scattering of the values requires further investigations. However, departure from unity observed even in the group with the values form 0.7 to 1.1 imply that the ratio $B \equiv 1$ implied by Ciz and Shapiro (2009), is not applicable for modelling of stress dependency of elastic properties of shales.

All four fitting parameters are plotted vs. the depth of the shale extraction in Figure 3. Crack orientation anisotropy parameter, $\eta$, reveals an exponential growth with the depth of origin (Figure 3a) which can be tracked easily in spite of the significant scattering of the fitting values. Higher values of $\eta$ mean better alignment of the cracks in the bedding plane; consequently, exponential increase of $\eta$ with the depth indicates fast growth of anisotropy of shale with increase of overburden pressure.

Tangential compliance of a single crack normalized on the area of the crack $B_{T}$ exponentially decreases with the depth (Figure 3b). This implies that in consort with expectations, cracks tend to be sufficiently stiffer in the shales that are recovered from larger depths that in the shales extracted from the shallower ones. The ratio of normal to tangential compliance, $B$, shows positive trend with the depth (Figure 3c), implying that normal stiffness of shales grows slower with the overburden than the tangential one. In other words with increase of the depth, cracks become relatively stiffer in the plane of the crack than in the normal direction. The characteristic pressure, $P_{0}$, shows no any obvious trend with the depth of origin (Figure 3d). $P_{0}$ is equal to $20 \mathrm{MPa}$ for the shallow and deep depth of less than $1500 \mathrm{~m}$ and more than $2500 \mathrm{~m}$. For the intermediate depth of $1500-2500 \mathrm{~m}, P_{0}$ drops to $10 \mathrm{MPa}$. While the facts that shales become stiffer and more anisotropic with depth are generally accepted, the increase of the ratio of normal to tangential compliance and the decrease of the characteristic pressure at the depth range of 1500-2500 m are new findings, which have to be explained.

The suggested model can be used for prediction of the unknown elastic parameters from the known ones. This problem is practically important either for laboratory measurements in shales, where $c_{13}$ 
constant is often unreliable or for field data analysis where log data give only two of five elastic constants of TI media. As the fitting problem described by equations (4)-(8) is over determined if the experimental data are obtained for several effective stresses, the four fitting parameters might be determined from experimental stress dependencies of an incomplete set of elastic constants. Then the fitting parameters can be used to predict stress dependencies of all five elastic constants of the TI medium.

For one of the shales, Figure 4 shows the results of prediction of stress dependencies of the elastic constants using as an input stress dependencies of five, three and two elastic constants. The predictions obtained for input of four elastic constants (c11, c33, c44, c66) are indistinguishable from those obtained for the input of five; the differences for other cases are small.

\section{Conclusions}

A new stress dependency model for TI media is developed and used to parameterise stress dependencies of about 20 shale elastic properties. The four fitting parameters (namely, tangential and normal compliances of a single discontinuity, characteristic pressure, and crack orientation anisotropy parameter) show obvious correlations with the depth from which the shale was extracted. With increase of the depth, the tangential compliance decreases exponentially and the ratio of normal to tangential compliance increases linearly. The crack orientation anisotropy parameter exponentially increases with the depth for the most of the shales indicating that cracks are getting more aligned in the bedding plane. The characteristic pressure shows no correlation with the extraction depth. The suggested model allows predicting of stress dependency of all five elastic constants if only two of them are known what can be used, for instance, for the reconstruction of all five elastic constants of shale from log data.

\section{References}

Ciz, R. and Shapiro, S.A. [2009] Stress-dependent anisotropy in transversely isotropic rocks: Comparison between theory and laboratory experiment on shale. Geophysics, 74(1), D7-D12.

Sayers, C. and Kachanov, M. [1995] Microcrack-indused elastic wave anisotropy of brittle rock, J. Geophys. Res., 100, 4149-4156.

Shapiro, C.A. and Kaselow, A. [2005] Porosity and elastic anisotropy of rocks under tectonic-stress and pore-pressure changes, Geophysics, 70(5), 27-38.

Wang, Z. [2002] Seismic anisotropy in sedimentary rocks, part 2: Laboratory data. Geophysics, 67(5), $1423-1440$.

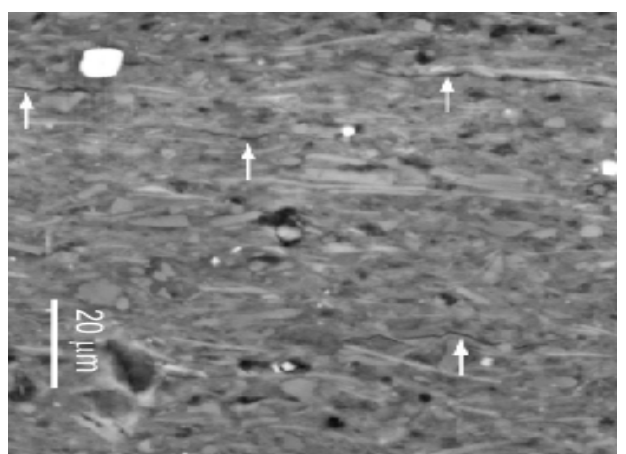

Figure 1 Office Basin shale shows presence of microfractures.

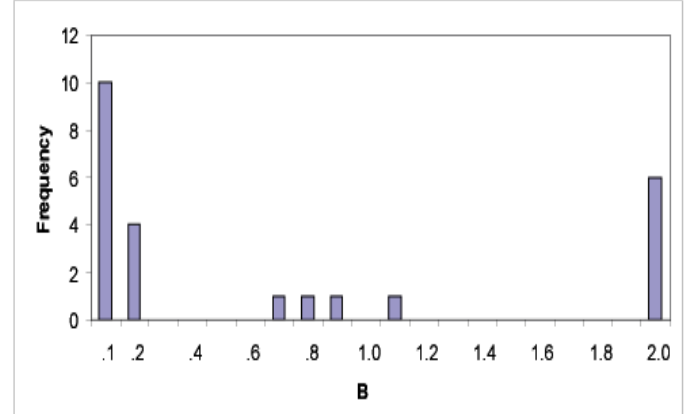

Figure 2 Histogram of the ratio of normal to tangential compliance for all the shale samples. Most of the values are far from unity. 


\section{Barcelona}

(a)

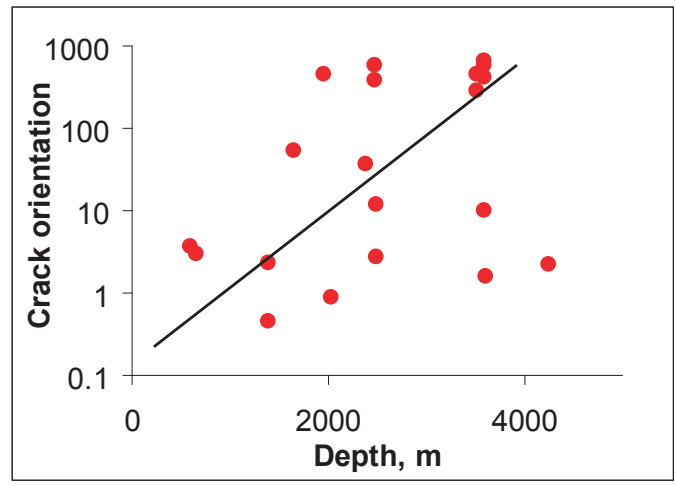

(c)

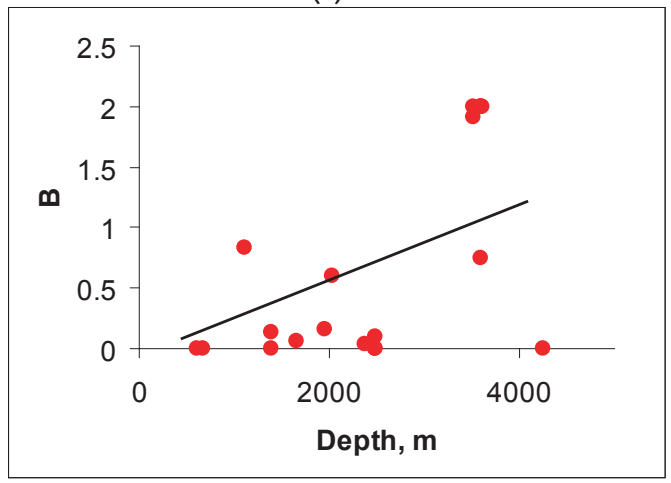

(b)

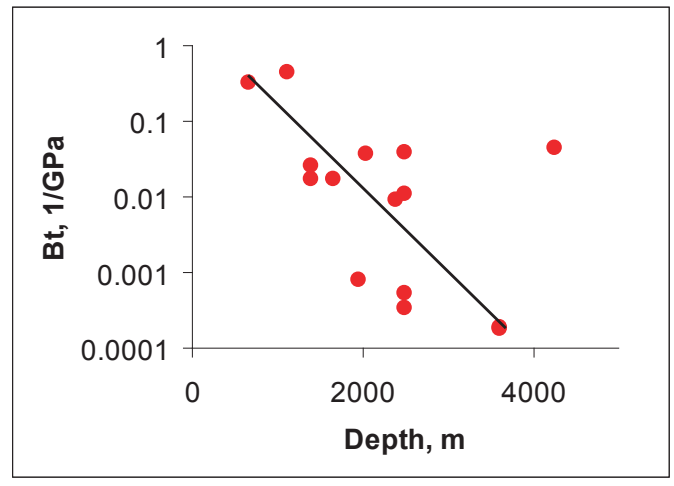

(d)

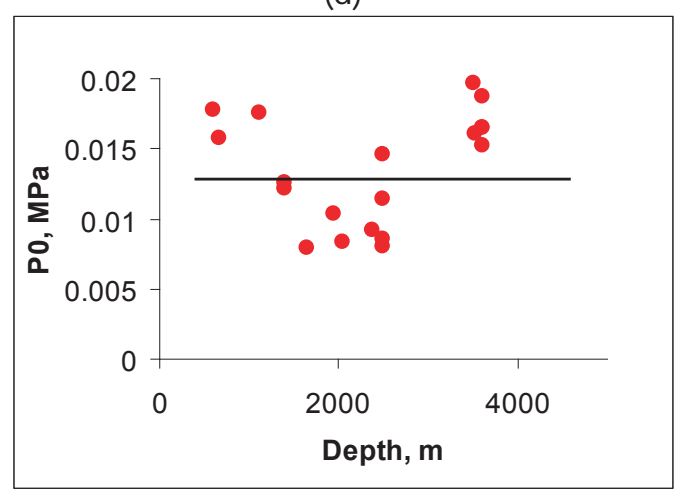

Figure 3 Variations with depth of (a) crack orientation distribution $\theta$, (b) tangential compliance $B_{T}$, (c) ratio of normal to tangential compliance $B,(d)$ characteristic pressure $P_{0}$.
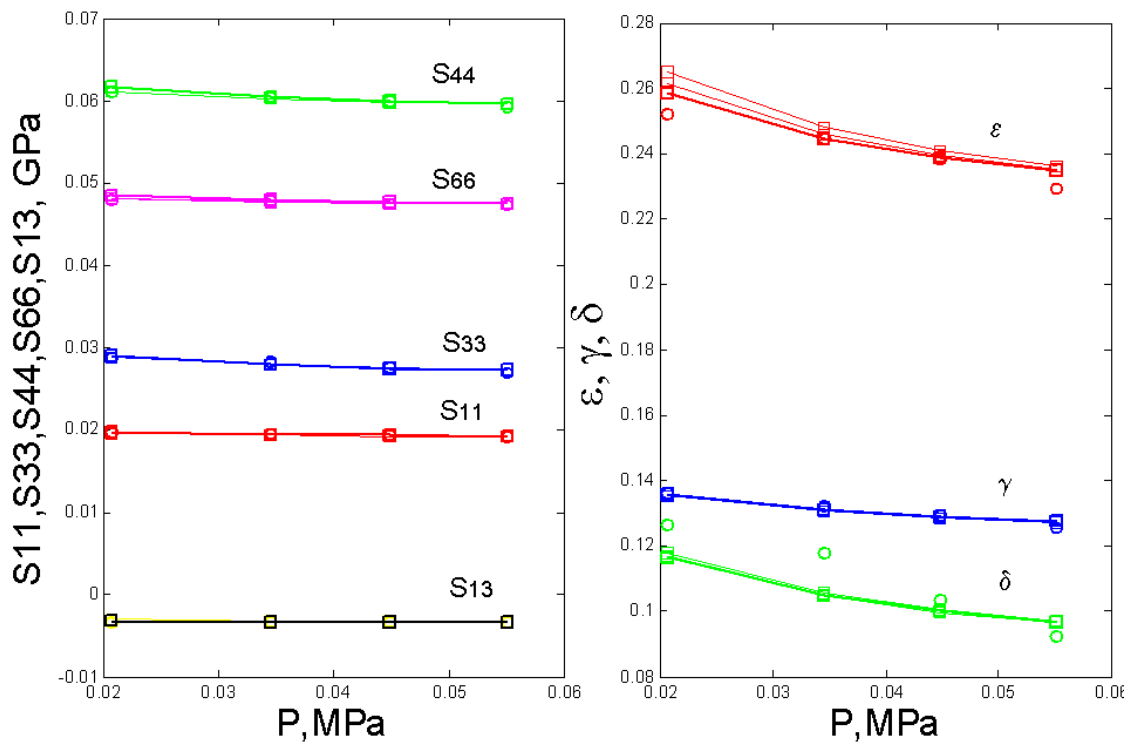

Figure 4 Compliances (left) and anisotropy parameters (right). Values calculated from ultrasonic measurements are shown by circles. Fits using full set of five compliances is shown by thick lines. Thin lines show fits using incomplete sets of compliances. In most of the cases the thin and thick lines coincide. 